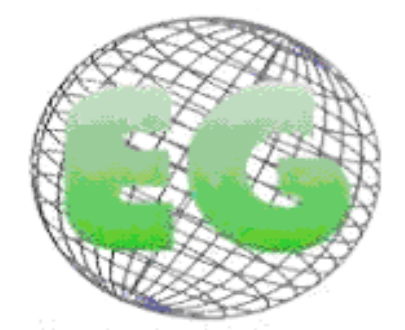

ISSN 1695-6141 N'25.

\title{
Fomento del autocuidado en la insuficiencia cardiaca
}

\section{Self-care promotion in heart failure}

\author{
*Olivella Fernández, M., Patricia Bonilla, C., Bastidas, CV. \\ *Mgs en Enfermería. Docentes Programa de Enfermería. Facultad de Ciencias de la Salud. Universidad del \\ Tolima-Ibagué. Colombia. \\ Palabras clave Insuficiencia cardiaca; autocuidado; cuidado de enfermería
}

Keywords: Self-care; heart failure; nursing care.

\section{RESUMEN}

Este artículo aborda analíticamente aspectos conceptuales del Modelo de Autocuidado de Orem, como estrategia que apunta a demostrar que el autocuidado es la forma más eficaz, pertinente, económica y oportuna para enfrentar la cronicidad y particularmente la insuficiencia cardiaca.

Fenómenos específicos como el envejecimiento poblacional y el uso de nuevas tecnologías, han repercutido en el aumento de la Insuficiencia Cardiaca.

El perfil epidemiológico del país muestra cómo las dos primeras causas de mortalidad, al sumarlas, superan tasas del $300 \%$, se refieren a eventos que afectan la salud cardiovascular. Esta alarmante situación lleva a reflexionar cómo la investigación disciplinar puede identificar lagunas en la literatura, en un tema específico y propio como es el autocuidado y formular recomendaciones para futuras investigaciones, que contribuyan al enfrentamiento de este flagelo

\section{ABSTRACT}

This articleapproaches the analytical conceptual aspects of Orem's Model, as a strategy to demonstrate that self-care is avery effective, pertinent, economic and opportune way to confront chronic diseases, particularly heart failure.

Specific phenomena like population aging and the use of new technologies have reverberated in the increase of the heart failure.

The epidemiological profile of the country shows that the first two reasons of mortality, which together total over300 \%, have to do with events that affect cardiovascular health. This alarming situation leads one to reflect on how research into this area may identify gaps in the literature, in a specific topic like self-care and to formulate recommendations for future investigations to counterthis scourge 


\section{AFRONTANDO LA INSUFICIENCIA CARDIACA: ¿TIENE ENFERMERÍA ALGO POR HACER?}

La Insuficiencia Cardíaca es frecuente en los países desarrollados y en todo el mundo ${ }^{(1)}$. La bibliografía reporta como una serie de síntomas que acompañan la enfermedad, constituyen el llamado "síndrome de anticipos", en donde el enfermo se enfrenta a cambios fisiológicos que incluyen entre otros, un ciclo de retención de líquidos, síntomas de una exacerbación aguda que generalmente culminan con una hospitalización. Todas estas situaciones podrían prevenirse con estrategias de autocuidado. ${ }^{(2)}$

Sin embargo, el auto-cuidado deficiente, sigue siendo extremadamente común en esta población de pacientes ${ }^{(2)}$. Menos de la mitad de los pacientes de Insuficiencia Cardiaca se pesan rutinariamente, para controlar la retención de líquidos ${ }^{(1)}$. Comer una dieta restringida en sodio es difícil para la mayoría de los pacientes, y los investigadores han documentado que poco más de la mitad de los pacientes con Insuficiencia Cardiaca tienen éxito en este aspecto del auto-cuidado.

Para la persona que vive en situación de cronicidad, resulta bastante difícil la adopción de un nuevo estilo de vida, desaprender conductas introyectadas a lo largo de la existencia y cambiarlas por nuevos hábitos, puede resultar fácil decirlo para el prestador de salud, pero para el enfermo y su familia constituyen un elemento desafiante. Es fundamental que el enfermo pueda darse cuenta de cuáles son los síntomas particulares que podrían inclusive llevarlo a una hospitalización, en el caso de la Insuficiencia Cardiaca, por ejemplo la dificultad para respirar, la hinchazón de los tobillos, la fatiga, pueden ser percibidos por el enfermo como malestares frecuentes y dejar de reconocerlos como síntomas agudos que requieren una pronta intervención ${ }^{(3)}$.

Es curioso observar que aunque el auto-cuidado es un elemento esencial y un componente integral para enfrentar la cronicidad y particularmente la Insuficiencia Cardiaca, es sorprendente que poco se sabe acerca de cómo aprenden los pacientes el auto-cuidado y los aspectos de su vida que promueven e inhiben su desempeño. Los investigadores han explorado una amplia variedad de factores como posibles predictores de auto-cuidado. Entre ellos se destacan: Factores de influencia del auto-cuidado en Insuficiencia Cardiaca incluyendo la misma patología ${ }^{(1)}$, el deterioro funcional, el deterioro de la cognición ${ }^{(4)}$, depresión $^{(1)}$, la excesiva somnolencia diurna ${ }^{(5)}$, y el apoyo familiar ${ }^{(3)}$.

El estudio más ilustrativo en relación con auto-cuidado en la Insuficiencia Cardiaca fue llevado a cabo por Horowitz, Rein, y Leventhal (2004), que en diversas entrevistas con pacientes de Insuficiencia Cardiaca, encontraron que comúnmente esta se percibe como una enfermedad aguda en lugar de una enfermedad crónica, convencidos de que la Insuficiencia Cardiaca, podía ser curada; cuando los síntomas se habían ido, los pacientes consideraban que su salud se había restablecido. Por lo tanto, en forma sutil e insidiosa los síntomas se iban exacerbando, sin que se haya reconocido el peligro. Como consecuencia de ello, los pacientes con síntomas mal vigilados, rara vez participan de un efectivo autocuidado. La mirada biomédica, predominante en muchos Sistemas de Salud del mundo, en cierta forma ayuda a esta creencia apoyando estas ideas erróneas, al no administrar citas especializadas y continuas a los pacientes con ausencia de síntomas.

Es esencial para el profesional de Enfermería entender en primera instancia el autocuidado, este se refiere a las conductas que los pacientes utilizan para mantener la estabilidad fisiológica como tomar la medicación y la respuesta a los síntomas cuando ocurren ${ }^{(6)}$. 
Enfermería particularmente encuentra un amplio marco teórico que direcciona el desarrollo disciplinar desde el abordaje de la Teoría de Autocuidado de Orem, esta se fundamenta en las premisas de que los individuos tienen la potencialidad para desarrollar sus habilidades intelectuales y prácticas y la motivación esencial para el autocuidado ${ }^{(7)}$. Orem parte de la premisa de que todo individuo está en capacidad de satisfacer su autocuidado.

El Modelo de Orem se fundamenta en el pilar de que los individuos tienen la potencialidad para desarrollar sus habilidades intelectuales y prácticas y la motivación esencial para el autocuidado ${ }^{(7)}$. La meta de enfermería dentro de esta perspectiva es empoderar a las personas para que satisfagan sus necesidades de autocuidado ayudándoles a desarrollar y ejercer sus capacidades de autocuidado (agencia). La teoría ofrece direcciones en el estudio de factores que condicionan el desarrollo, la operacionalidad y la calidad del autocuidado ejecutado. La inclusión de la orientación sociocultural como uno de los factores básicos condicionantes amplían la generalidad de la teoría y a la vez su utilidad global. ${ }^{(8)}$

Un factor determinante para llevar a cabo las recomendaciones terapéuticas en las personas con Insuficiencia Cardiaca es la capacidad de autocuidado, según Orem las capacidades de autocuidado "se refieren a la capacidad humana de los individuos para realizar acciones con el objeto de cuidar de sí mismos y de otros".

Se ha escrito mucho sobre la importancia del auto-cuidado en la Insuficiencia Cardiaca, pero los investigadores siguen perplejos acerca de ¿por qué algunos pacientes desarrollan en forma maestra sus habilidades de auto-cuidado y otras no?, este es sin duda un campo fructífero para el desarrollo disciplinar de la Enfermería.

Los esfuerzos para reforzar el auto-cuidado en la insuficiencia cardiaca de los pacientes son de importancia primordial para la mejora de conductas relacionadas con la dieta y adhesión a la medicación, la reducción de la hospitalización y la mejora global de resultados. El Autocuidado en la insuficiencia cardíaca es muy variable, y los nuevos enfoques son necesarios para promover las hospitalizaciones evitables, reducir los síntomas y mejorar la calidad de vida. La publicación de guías de práctica clínica sugieren que los pacientes con insuficiencia cardiaca congestiva y los miembros de su familia o cuidadores deben recibir educación individualizada y el asesoramiento que hace hincapié en el auto-cuidado; sin embargo, los datos para orientar la educación familiar y atención en la insuficiencia cardíaca son escasos $^{(3)}$.

\section{ELEMENTOS PARA LA DISCUSIÓN}

La Insuficiencia cardiaca es una realidad que permea las tasas de mortalidad y morbilidad de todos los lugares del planeta, el uso de los recursos sanitarios, después del diagnóstico, siguen generando altos costos a pesar de las nuevas tecnologías y nuevas terapias. Los pacientes a menudo tienen limitaciones significativas en múltiples áreas, incluyendo el desarrollo físico, cognitivo, emocional, funcionamiento social, salud y calidad de vida, en general. ${ }^{(9)}$

El conocimiento de los efectos de la Insuficiencia Cardiaca y su tratamiento muestran importantes resultados que dan orientaciones para el desarrollo de intervenciones, por parte de los profesionales de la salud. Muchas de estas intervenciones son sensibles a la práctica enfermera es decir, estarán parcial o totalmente influenciadas por el cuidado de enfermería ${ }^{(10)}$ 
En este sentido, particularmente para Colombia, es prioritario identificar y describir las investigaciones recientes que se centren en el cuidado de enfermería transformador, que muestre resultados sensibles de estrategias que fomenten el autocuidado en personas que viven en situación de cronicidad, específicamente enfrentando la Insuficiencia Cardiaca; lastimosamente el estado del arte al respecto, no muestra grandes resultados.

Los avances en la investigación en América Latina en el tema del autocuidado y la Insuficiencia Cardiaca apoyan lo que investigadores han demostrado en otros contextos, que la bibliografía propia de enfermería que reporte intervenciones y mediciones del autocuidado en personas con Insuficiencia Cardiaca, no es suficiente. El hecho de que en sólo uno de cada 10 pacientes con Insuficiencia Cardiaca se pueda esperar que domine su auto-cuidado ilustra la importancia de encontrar maneras para desarrollar investigación al respecto ${ }^{(3)}$. Causa gran preocupación el hecho de que inclusive en personas hospitalizadas una serie de acciones que comprenden el autocuidado diario de una persona con Insuficiencia Cardiaca, no sea tenido en cuenta. Un estudio reciente en el Brasil con pacientes descompensados internados en un servicio de emergencia en donde se incluyeron 256 pacientes, con edad promedio de $63 \pm 13$ años, mostró en cuanto a la realización de los cuidados no farmacológicos como restricción de sal, restricción hídrica, control de diuresis y control de peso, se identificó que existe una contradicción entre lo que es prescrito y lo que es efectivamente ejecutado. Se demostró que esos cuidados no fueron realizados en su totalidad por el equipo de enfermería ${ }^{(11)}$

\section{CONSIDERACIONES FINALES}

El autocuidado es una conducta aprendida, sin embargo, conocer las medidas de autocuidado no garantiza que estas se lleven a cabo. Un reciente estudio muestra cómo no todos los profesionales de enfermería estudiados abordan con claridad la importancia del autocuidado cuando se argumenta que, para cuidar del otro, antes es necesario cuidar de sí mismo, pues el cuidado solamente ocurre con la transmisión de los sentimientos y potencialidades personales a la otra persona ${ }^{(12)}$

Se podría decir que existe un vacío conceptual entre aprender el autocuidado y aprehender el autocuidado, en el sentido de que este último signifique una interiorización tan profunda del mismo que conlleve a cambios de vida, para el caso de la Insuficiencia Cardiaca a realizar esa serie de acciones diarias en forma rutinaria que constituyan en el día a día hacer realidad la acción de cuidar de sí mismo. Investigaciones que reporten la eficacia del autocuidado en enfermedades como la Insuficiencia Cardiaca constituyen un campo fructífero y un aporte fundamental de la Enfermería en el afrontamiento de este flagelo de orden mundial.

\section{BIBLIOGRAFÍA}

(1). Moser MK, Watkins JF. Conceptualizing self-care in heart failure: a life course model of patient characteristics. J Cardiovasc Nurs. 2008;23:205-218.

(2). Bennett SJ, Baker SL, Huster GA. Quality of life in women with heart failure. Health Care Women Int. 1998;19: 217-229.

(3). Riegel, Barbara; Vaughan Dickson, Victoria; G Nursing Lee R.; Deatrick, Janet A.Factors Associated With the Development of Expertise in Heart Failure Self-Care. Research. 56(4):235-243, July/August 2007.oldberg.

(4). Assessment of cognitive function in heart failure patients www.ncbi.nlm.nih.gov/pubmed/16359923. 
(5). Factors Associated with the development of expertise in heart failure self-care journals.lww.com/.../ factors_associated_with_the...

(6). Riegel, B., Carlson, B., Moser, D.K. Psychometric testing of the self-care of heart failure index. journal of cardiac failure, 200410 (4), 350-360.

(7). Orem. Dorothea E. Modelo de Orem: Conceptos de Enfermería en la Práctica. Ediciones Científicas y Técnicas, S.A. Barcelona, 1993. p. 163.

(8). Parker, Marilyn. Para avanzar la ciencia de enfermería y la práctica profesional. En Nursing theories and nursing practice, 2002, f.a. Davis co hiladelphia: capítulo 13. pág. 1. resumen del capítulo por Edilma de Reales, noviembre 2001.p. 13.

(9). Grady, Kathleen L. PhD, RN, APN, FAAN. Self-care and Quality of Life Outcomes in Heart Failure Patients. Journal of Cardiovascular Nursing. 23(3):285-292, May/June 2008.

(10). Deaton, Christi, PhD, RN, Grady, Kathleen, PhD, RN, FAAN, FAH. State of the Science for Cardiovascular Nursing Outcomes: Heart Failure. Journal of Cardiovascular Nursing. 19(5):329-338, September/October 2004.

(11). Linhares JC, Aliti GB, Castro RA, Rabelo ER. Prescripción y realización del manejo nofarmacológico para pacientes con insuficiencia cardíaca descompensada. Rev. Latino-

Am. Enfermagem [Internet]. nov.-dec. 2010 [acceso en http://www.scielo.br/pdf/rlae/v18n6/es 15.pdf

(12). Hernández Vergel Lázaro Luis, Zequeira Betancourt Dulce Maria, Miranda Guerra Amauri de Jesús. La percepción del cuidado en profesionales de enfermería. Rev Cubana Enfermer [revista en la Internet]. 2010 Mar [citado 2011 Jul 19] ; 26(1): 30-41. Disponible en: http://scielo.sld.cu/scielo.php?script=sci arttext\&pid=S0864-

$\underline{03192010000100007 \& \operatorname{lng}=\mathrm{es}}$.

ISSN 1695-6141

(๖) COPYRIGHT Servicio de Publicaciones - Universidad de Murcia 